\title{
Leveraging multi-modal user-labeled data for improved accuracy in interpretation of ECG recordings
}

\author{
Maharjan, Raju; Bækgaard, Per; Bardram, Jakob E.
}

\section{Published in:}

Proceedings of the 2018 ACM International Joint Conference and 2018 International Symposium on Pervasive and Ubiquitous Computing and Wearable Computers

Link to article, DOI:

$10.1145 / 3267305.3267548$

Publication date:

2018

Document Version

Early version, also known as pre-print

Link back to DTU Orbit

Citation (APA):

Maharjan, R., Bækgaard, P., \& Bardram, J. E. (2018). Leveraging multi-modal user-labeled data for improved accuracy in interpretation of ECG recordings. In Proceedings of the 2018 ACM International Joint Conference and 2018 International Symposium on Pervasive and Ubiquitous Computing and Wearable Computers (pp. 636641). Association for Computing Machinery. https://doi.org/10.1145/3267305.3267548

\section{General rights}

Copyright and moral rights for the publications made accessible in the public portal are retained by the authors and/or other copyright owners and it is a condition of accessing publications that users recognise and abide by the legal requirements associated with these rights.

- Users may download and print one copy of any publication from the public portal for the purpose of private study or research.

- You may not further distribute the material or use it for any profit-making activity or commercial gain

- You may freely distribute the URL identifying the publication in the public portal 


\section{Leveraging Multi-modal User-labeled Data for Improved Accuracy in Interpretation of ECG Recordings}

Raju Maharjan,

Per Bækgaard,

Jakob E. Bardram

Copenhagen Center for Health

Technology

Technical University of Denmark

Lyngby, Denmark

\{rajm,pgba,jakba\}@dtu.dk
Permission to make digital or hard copies of all or part of this work for personal or classroom use is granted without fee provided that copies are not made or distributed for profft or commercial advantage and that copies bear this notice and the full citation on the first page. Copyrights for components of this work owned by others than ACM must be honod. Abstractig wh chedis is pernited. To copy otherwise, or republish to post on servers or to redistribute to lists, requires prior specific permission and/or

pore, Singapore.

源

https://doi.org/10.1145/3267305.3267548

\begin{abstract}
This paper presents our preliminary design of the Reaching the Frail Elderly Patient for Optimizing Diagnosis of Atrial Fibrillation (REAFEL) system that helps to improve accuracy in interpretation of Electrocardiography (ECG) recordings by leveraging multi-modal user-labeled data and other contextual information from mobile devices. We describe the methods to collect and visualize the data, discuss the challenges associated with the project and conclude the paper by outlining future work.
\end{abstract}

\section{Author Keywords}

Personal Health Technology; mHealth; User-labeled data; Patient Reported Outcomes

\section{ACM Classification Keywords}

H.5.m [Information interfaces and presentation (e.g., $\mathrm{HCl})$ ]:

Miscellaneous.

\section{Introduction}

Heart related diseases are the most common causes of death worldwide [16]. The Electrocardiography (ECG) is a standard, low-cost, noninvasive, and effective tool for diagnosis and classification of heart rhythm disorders, also known as arrhythmias [4]. A number of systems have previously been developed using different techniques to record, auto-detect and classify heart rhythm 


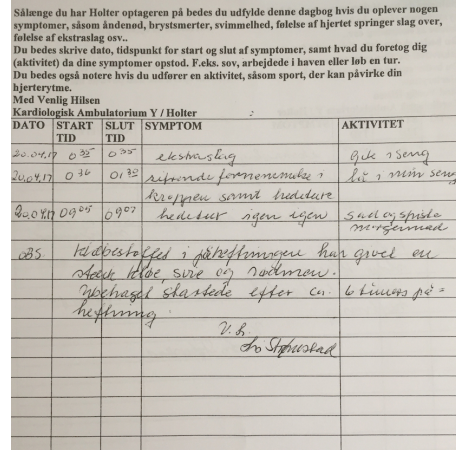

Figure 1: Patient journal disorders $[18,8,12]$. Often these systems are inaccurate and flawed due to misinterpretation and misclassification of heart rhythm disorders [1, 3]. Some studies have indicated that continuous monitoring of ECG with contextual information is beneficial to improve accuracy in interpretation of heart rhythm disorders and prediction of heart related diseases [11, 13]

On the one hand, research has focused on creating robust, context-aware and intelligent systems and have shown the value of the patient's physical activity and other contextual information from smartphone sensors to improve monitoring, detection, and classification of heart rhythm disorders [11, 2, 13, 7]. On the other hand, studies have supported the need for assessment of user-labeled data (ULD) such as Patient reported outcome (PRO) ${ }^{1}$ in heart related disease management $[14,5]$ because PROs can provide a better representation of a patient's health status by "providing meaningful information that complements other clinical and physiological information or, in appropriate cases, be used in lieu of clinical and physiological endpoints" [15]. However, limited research has been done to leverage PROs and other contextual information from smartphone sensors with ECG data for better prediction and classification of heart rhythm disorders.

In this context, we are designing a system called Reaching the Frail Elderly Patient for Optimizing Diagnosis of Atrial Fibrillation (REAFEL) ${ }^{2}$ that collects, processes and visualizes ECG, contextual, and PRO data from the patient. The collected data will provide context and completeness to

${ }^{1}$ Patient reported outcome (PRO) is defined as any report of the status of a patient's health condition that comes directly from the patient, without interpretation of the patient's response by a clinician or anyone else [5]

${ }^{2}$ http://www.cachet.dk/research/ research-projects/reafel help improve accuracy in interpretation of ECG data for early detection of heart rhythm disorders.

\section{Preliminary Design}

To understand how the ECG data can be contextualized and made easy to interpret, we conducted a user-centered design process at a large Danish University Hospital in Copenhagen. Participants in the meetings were cardiologists, electro-physiologist, nurses, medical interns UX designers, and computer scientists. We explored the systems they are currently using to collect and analyze the ECG data.

Based on these observations and design meetings, an initial outline of the system was drawn, as shown in Figure 2. The system has two domains of data collection:

- Multi-modal ULD - Event marks and PRO from the ECG recording device and smart-phone application (Figure 2-A)

- Contextual information - Physical activities (walking, running, cycling etc.) and physical position (sleeping, standing, sitting etc.) from the sensors in the smartphone and the ECG device (Figure 2-B)

We have incorporated the above mentioned domains to design the proposed system which aims to contribute towards the improved accuracy of ECG data interpretation. Each of the domains and their implementation in the system is explained below.

Multi-modal user-labeled data (ULD)

Although the use of PRO in the management of heart condition is common in clinical settings and have been considered valuable to contextualize the ECG data, it is underutilized and has better potential in interpretation and understanding of the patient's heart condition [14]. Currently 


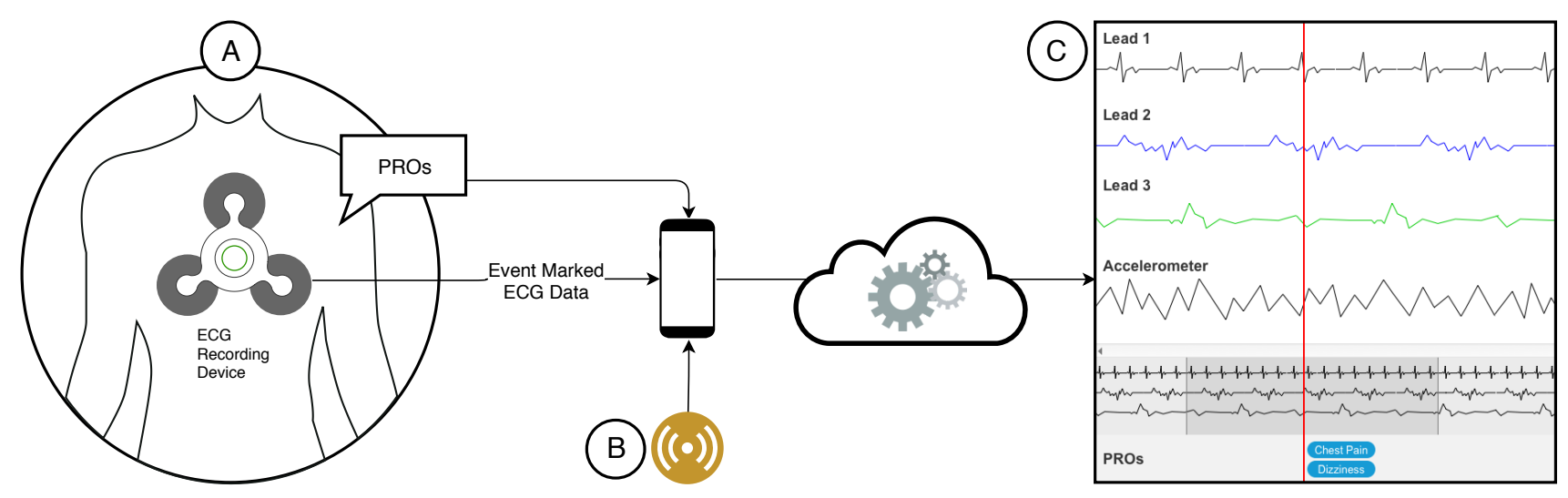

Figure 2: REAFEL system overview - (A) User-labeled data: event marked ECG data and PRO (B) Contextual information such as physical activity (walking, running, cycling etc.) from smart-phone sensors (C) ECG data visualization with contextual information.

in the hospital, PRO data from patients are collected using paper-based diaries, as shown in Figure 1. However, the clinicians stated that they could not rely on the PRO as they may be inaccurate and flawed because they are often based on patient recalls and not in situ, i.e., they do not represent a valid ecological momentary assessment (EMA) [10]

To address this issue, the proposed system will allow collection of event marks on ECG data and PRO in situ. Patients can press the button on the ECG recording device when they experience any symptoms. This creates a mark on the ECG data and prompts the patient to enter more details regarding the event via the smartphone application. The clinicians can view this information along with other contextual data from smartphone sensors, which will help to improve accuracy in interpretation of the ECG data.

\section{Contextual information}

Patient's physical activity such as walking, running, biking, etc. influence ECG data and often decrease its diagnostic quality due to motion artifacts [6]. In the current system, clinicians often have to guess certain patterns of motion artifacts based on their experience and expertise.

The cardiologist stated that physical activity of the patient is an important information to complement and interpret certain patterns of ECG data. The cardiologist gave an example of a telemetric ECG monitoring scenario where the ECG device stops sending the ECG signals because the device might have been unmounted, which could be interpreted in many ways.

In the current design, the following contextual parameters have been included by the cardiologist as relevant information in monitoring and diagnosis of heart-related episodes.

- Accelerometer and gyroscope data from the ECG device to determine the position of the patient (standing, sitting, laying down). 
- Location, step counts, physical activity (e.g. walking, biking, running etc.), sleep, and external temperature from the smartphone.

This kind of data can help understand the state of the device and the patient, as well as his or her activity, sleep patterns, and surrounding environment, which is an important information to contextualize and interpret certain patterns of ECG data.

\section{Discussion}

In our design, we have identified a set of challenges in collection and visualization of multi-modal ULD and contextual information from the ECG device and smartphone sensors.

\section{Collecting PRO in situ}

Collecting PRO in situ may not be feasible in cases where the patients experience severe symptoms and suffer from associated physical limitations. For example, if the patient is dizzy and is about to faint, it is unlikely that the patient will be able to enter the PRO in the smartphone application. An alternative could be to allow patients to enter PRO for the marked events at a later time when they have recovered from any physical distress. However, this will overrule the idea of collecting multi-modal ULD in situ to avoid inaccuracy due to patient recalls. In the workshop, we would like to discuss better approaches in collecting ULD in situ.

To avoid manual entry of the PRO data, the smartphone application could start recording the patient's voice as soon as the event marker button is pressed. Alternatively, an algorithm could be developed to automatically start recording as soon as it detects a certain pattern of rhythm disorder. Nevertheless, both of these solutions raise privacy concerns because the smartphone application may record sound from the surroundings as well. Therefore, we would like to discuss the privacy issues and the solution in the process of data collection.

Visualizing ECG data with contextual information An ECG recording device collects $60-100$ heart beats per minute for an adult with a normal resting heart rate [9]. Analyzing large sets of ECG recordings is a challenge in itself [17]. Inclusion of PRO and other contextual information from smartphone sensors will add complexity to this. To the best of our knowledge, no previous study has investigated visualization of ECG data with PRO and contextual information to improve interpretation of ECG data. Any input and suggestion on our preliminary design will be much appreciated.

\section{Conclusion and Future Work}

To achieve the primary objective of the REAFEL project, we are designing a robust system that will help improve accuracy in interpretation of ECG recordings by collecting and visualizing multi-modal user labeled data (event marks and PRO) and other contextual information (patient's physical activities such as walking, running, cycling etc.) from smartphone devices.

Additional research is required to determine if any other information such as temperature, air pressure, humidity etc. collected via smartphone sensors are clinically relevant to improve accuracy in ECG data interpretation. Research is also needed to find the best way to visualize the heterogeneous data including PRO and other contextual information in a meaningful way so that it can help clinicians better interpret the ECG recordings.

We aim to implement a prototype of the system in a clinical setting and run a feasibility study. The study will recruit $\mathrm{N}=50$ patients and 10 clinicians over the course of 12 months. This feasibility study aims to investigate system 
usability as well as clinical usefulness in monitoring and diagnosis of heart-related diseases.

\section{Acknowledgements}

The REAFEL project is funded by the Innovation Fund Denmark.

\section{References}

[1] Anh, D., Krishnan, S., and Bogun, F. Accuracy of electrocardiogram interpretation by cardiologists in the setting of incorrect computer analysis. Journal of electrocardiology 39, 3 (2006), 343-345.

[2] Arif, M., et al. Robust electrocardiogram (ecg) beat classification using discrete wavelet transform. Physiological measurement 29, 5 (2008), 555.

[3] Bogun, F., Anh, D., and Kalahasty, G. Misdiagnosis of atrial fibrillation and its clinical consequences. ACC Current Journal Review 2, 14 (2005), 59-60.

[4] De Chazal, P., O'Dwyer, M., and Reilly, R. B. Automatic classification of heartbeats using ecg morphology and heartbeat interval features. IEEE transactions on biomedical engineering 51, 7 (2004), 1196-1206.

[5] Group, F.-N. B. W., et al. Best (biomarkers, endpoints, and other tools) resource.

[6] Han, D., Hong, J., Shin, J., and Lee, T. Accelerometer based motion noise analysis of ecg signal. In World Congress on Medical Physics and Biomedical Engineering, September 7-12, 2009, Munich, Germany, Springer (2009), 198-201.

[7] Healey, J., and Logan, B. Wearable wellness monitoring using ecg and accelerometer data. In Wearable Computers, 2005. Proceedings. Ninth IEEE International Symposium on, IEEE (2005), 220-221.

[8] Hu, Y. H., Palreddy, S., and Tompkins, W. J. A patient-adaptable ecg beat classifier using a mixture of experts approach. IEEE transactions on biomedical engineering 44, 9 (1997), 891-900.

[9] January, C. T., Wann, L. S., Alpert, J. S., Calkins, H., Cigarroa, J. E., Conti, J. B., Ellinor, P. T., Ezekowitz, M. D., Field, M. E., Murray, K. T., et al. 2014 aha/acc/hrs guideline for the management of patients with atrial fibrillation: a report of the american college of cardiology/american heart association task force on practice guidelines and the heart rhythm society. Journal of the American College of Cardiology 64, 21 (2014), e1-e76.

[10] McKeon, A., McCue, M., Skidmore, E., Schein, M., and Kulzer, J. Ecological momentary assessment for rehabilitation of chronic illness and disability. Disability and rehabilitation 40, 8 (2018), 974-987.

[11] Miao, F., Cheng, Y., He, Y., He, Q., and Li, Y. A wearable context-aware ecg monitoring system integrated with built-in kinematic sensors of the smartphone. Sensors 15, 5 (2015), 11465-11484.

[12] Nasiri, J. A., Naghibzadeh, M., Yazdi, H. S., and Naghibzadeh, B. Ecg arrhythmia classification with support vector machines and genetic algorithm. In Computer Modeling and Simulation, 2009. EMS'09. Third UKSim European Symposium on, IEEE (2009), 187-192.

[13] Spadini, F., Vergari, F., Nachman, L., Lamberti, C., and Cinotti, T. S. A wireless and context-aware ecg monitor: An imote2 based portable system. In Computers in Cardiology, 2008, IEEE (2008), 997-1000.

[14] Thompson, L. E., Bekelman, D. B., Allen, L. A., and Peterson, P. N. Patient-reported outcomes in heart failure: existing measures and future uses. Current heart failure reports 12, 3 (2015), 236-246. 
[15] U.S. Department of Health and Human Services. Value and use of patient reported outcomes (pros) in assessing effects of medical devices, cdrh strategic priorities 2016-2017.

[16] World Health Organization. Prevention of cardiovascular disease. World Health Organization, 2007.

[17] Xu, K., Guo, S., Cao, N., Gotz, D., Xu, A., Qu, H., Yao, Z., and Chen, Y. Ecglens: Interactive visual exploration of large scale ecg data for arrhythmia detection. In Proceedings of the $2018 \mathrm{CHI}$ Conference on Human Factors in Computing Systems, ACM (2018), 663.

[18] Yeap, T., Johnson, F., and Rachniowski, M. Ecg beat classification by a neural network. In Engineering in Medicine and Biology Society, 1990., Proceedings of the Twelfth Annual International Conference of the IEEE, IEEE (1990), 1457-1458. 